Service social

\title{
Adoption « ouverte » : quelques enjeux et constats
}

\section{Dominique Goubau et Suzanne Beaudoin}

Volume 45, numéro 2, 1996

Droit et pratiques sociales

URI : https://id.erudit.org/iderudit/706726ar

DOI : https://doi.org/10.7202/706726ar

Aller au sommaire du numéro

Éditeur(s)

École de service social de l'Université Laval

ISSN

1708-1734 (numérique)

Découvrir la revue

Citer cet article

Goubau, D. \& Beaudoin, S. (1996). Adoption « ouverte » : quelques enjeux et constats. Service social, 45(2), 51-72. https://doi.org/10.7202/706726ar

\section{Résumé de l'article}

L'adoption ouverte est un phénomène relativement récent au Québec et qui est peu documenté. S'agit-il d'un nouveau modèle d'adoption ou tout simplement d'une variante dans le processus clinique qui aboutit à l'adoption sans pour autant remettre en question le modèle traditionnel de ce transfert familial ? Le présent article expose quelques enjeux de l'adoption ouverte, à la lumière des premiers résultats d'une recherche empirique sur cette question. d'utilisation que vous pouvez consulter en ligne.

https://apropos.erudit.org/fr/usagers/politique-dutilisation/ 


\title{
Adoption "ouverte»: quelques enjeux et constats
}

\author{
Dominique GOUBAU \\ Professeur \\ Faculté de droit, Université Laval \\ Suzanne BEAUDOIN \\ Chercheuse, Centres jeunesse de Québec \\ Membre du Centre de recherche sur les services \\ communautaires, Université Laval
}

\section{INTRODUCTION}

Depuis quelques années, on entend parler de plus en plus du concept d' «adoption ouverte", par opposition à l'adoption fermée ou traditionnelle. Bien connue des intervenants sociaux, cette notion assez récente n'a, jusqu'à ce jour, pas attiré spécialement l'attention des chercheurs au Québec, alors qu'aux États-Unis les études sur cette question se multiplient depuis près de dix ans. Il faut dire que chez nos voisins du Sud l'adoption ouverte est courante depuis le début des années 1970.

Nous remercions vivement Claire O'Neil et Isabelle Roy pour leur précieux travail d'auxiliaires de recherche. Ce projet bénéficie du financement du Conseil de la recherche en sciences humaines du Canada (CRSHC) et de la Fondation de la recherche du Barreau du Québec. 
Il semblerait d'ailleurs que dans la majorité des services d'adoption aux États-Unis les parents adoptifs ont une forme quelconque de contact avec les parents biologiques (généralement avec la mère biologique) ${ }^{1}$. Le présent texte fait suite à un projet de recherche sur l'adoption ouverte au Québec et entend présenter la partie des résultats concernant l'analyse de 30 dossiers, c'està-dire la quasi-totalité des adoptions ouvertes de la région de Québec pour la période de janvier 1993 à juin 1995. Après avoir situé la question de l'adoption ouverte, nous ferons état de certains enjeux qu'elle comporte. Dans un article à venir, nous aurons l'occasion d'analyser et de comparer les pratiques d'ouverture dans six Centres jeunesse de la province.

\section{LE MODÈLE TRADITIONNEL: DE L'ADOPTION FERMÉE À L'ADOPTION ENTROUVERTE}

L'adoption traditionnelle, dite fermée, repose essentiellement sur le principe de l'effacement de la famille d'origine au profit de la famille adoptive. C'est le modèle qui fut retenu dans la quasi-totalité des pays occidentaux lorsque, à la fin du XIX et surtout au début du $\mathrm{XX}^{\mathrm{e}}$ siècle, furent élaborées les premières législations en la matière ${ }^{2}$. Ce fut tout naturellement ce modèle que le Québec privilégia dans la première loi sur l'adoption en 1924. Le modèle traditionnel propose une forme unique d'adoption par laquelle l'enfant adopté devient, à tous égards, le fils ou la fille des parents adoptants. Les parents biologiques, par le seul fait du jugement d'adoption, perdent alors leur statut de parents pour devenir, juridiquement parlant, des étrangers à l'enfant. La création d'un nouveau lien et la rupture complète du lien de filiation biologique, caractéristiques premières du modèle traditionnel, sont généralement complétées par une procédure dont le caractère confidentiel a pour but de préserver l'anonymat des parties et d'imposer le secret autour des actes de naissance d'origine ainsi que des dossiers d'adoption.

Fiction du droit, le modèle traditionnel pur s'articule ainsi autour de trois concepts fondateurs : rupture, confidentialité et secret. Le présent article n'est pas le lieu pour s'interroger sur les raisons profondes qui ont mené à la création du modèle traditionnel et qu'il faut situer, notamment, dans le contexte historique des stigmates imposés socialement et juridiquement au statut d'enfant illégitime ou bâtard. Soulignons simplement 
qu'indépendamment des finalités de l'institution, d'ailleurs fluctuantes dans le temps (par exemple: assurer une descendance aux adultes infertiles, garantir la pérennité du patrimoine par la présence d'un héritier, donner un statut à l'enfant abandonné, protéger l'enfant en danger), il est clair que l'adoption traditionnelle part du principe selon lequel l'évacuation la plus complète et la plus rapide possible de la famille d'origine constitue une condition de réussite de l'opération. La famille adoptive est, dès lors, une famille de substitution. La version moderne de cette façon de voir les choses consiste à dire que l'exclusivité dans l'appartenance familiale (et par conséquent l'exclusion des liens avec la famille d'origine) est de l'essence même de l'institution parce qu'elle va dans le sens du meilleur intérêt de l'enfant, seule justification acceptable - et avouable ${ }^{3}$ - de l'adoption contemporaine.

La dimension «secret» du modèle traditionnel fut considérablement nuancée, notamment sous l'impulsion des travaux de David Kirk qui, déjà en 1960, défendait l'idée (aujourd'hui unanimement acceptée) qu'il est important de reconnaître l'enfant adopté dans sa spécificité et donc dans sa différence ${ }^{4}$. L'idée de secret a aussi été fortement ébranlée dans les années 1970 et 1980 à l'occasion des pressions exercées par le mouvement social en faveur du droit aux origines. De nombreuses études scientifiques avaient alors mis au jour les effets néfastes potentiels de la confidentialité absolue ${ }^{5}$. Comme on le sait, ces pressions ont ultimement abouti, dans la plupart des pays occidentaux comme au Québec, à la mise en place de normes juridiques visant à encadrer (c'est-à-dire à permettre et à contrôler) les retrouvailles entre adoptés et parents d'origine, tout en essayant de maintenir un équilibre entre le principe du droit de connaître ses origines et celui du droit au respect de la vie privée. $\mathrm{Au}$ concept de secret fut opposé celui d'une relative transparence.

Cependant, là ne s'arrête pas la demande actuelle d'ouverture qui participe d'une profonde remise en question de l'institution $^{6}$. Désormais, les deux autres dimensions du modèle traditionnel - rupture et confidentialité - sont aussi remises en question par la pratique quotidienne de l'adoption dans plusieurs pays, comme l'Angleterre, la Nouvelle-Zélande, l'Australie, les États-Unis ${ }^{7}$. Ainsi, il est clair qu'aujourd'hui de nombreuses femmes, qui envisagent la solution de l'adoption pour leur enfant (né ou à venir), entendent participer plus activement au choix des futurs parents adoptifs. Certaines 
d'entre elles vont exiger d'avoir au moins une rencontre préalable avec les «candidats».

Nous avons même pu constater que, dans quelques cas, cette rencontre prend des allures de véritable examen de passage... Le consentement à l'adoption se trouve donc régulièrement conditionné par des exigences de plus en plus précises de la part des mères biologiques. De même, des candidats à l'adoption se trouvent placés devant le fait qu'ils pourraient bien devoir accepter l'idée d'un certain maintien de contact entre leur futur enfant et sa famille d'origine. En d'autres mots, la pratique sociale semble à première vue s'être considérablement écartée du modèle traditionnel, tel que proposé par les normes juridiques.

Il convient toutefois de nuancer quelque peu l'affirmation selon laquelle l'adoption, telle qu'elle est prévue par les normes légales actuelles, est de type complètement fermé. En effet, il existe déjà dans la législation du Québec des éléments non négligeables d'ouverture dans le processus. Nous avons déjà évoqué le plus important d'entre eux, soit la possibilité des retrouvailles post-adoption ${ }^{8}$. Le «consentement spécial» en constitue un autre: en principe le parent biologique ne peut choisir les parents adoptifs; il peut cependant le faire (et donc donner un consentement spécial) lorsqu'il entend confier son enfant à un très proche parent ${ }^{9}$. La loi reconnaît donc l'adoption privée dans la famille restreinte. Mentionnons également que la législation autorise expressément la transmission de sommaires (anonymes, il est vrai) des antécédents de l'enfant et même des parents adoptifs ${ }^{10}$. Ces antécédents sont parfois très détaillés et constituent indéniablement une «ouverture», un trait d'union entre les deux familles. Finalement, on peut noter que la loi invite le directeur de la Protection de la jeunesse à permettre aux parents de faire entendre leurs points de vue et d'exprimer leurs préoccupations ${ }^{11}$, ce qui comporte en soi le ferment d'une certaine ouverture dans le processus administratif. Cependant, malgré ces quelques éléments, il faut bien constater que l'adoption légale demeure encore et toujours essentiellement de type fermé et confidentiel ayant pour effet principal la rupture totale des liens entre l'enfant et sa première famille. Ce modèle n'assigne aucun rôle particulier au parent d'origine si ce n'est celui de consentir à l'adoption. De plus, il s'agit d'un modèle qui, par définition, propose une conception qui cantonne l'adoption à un moment bien précis dans le temps (en l'occurrence le jugement d'adoption) plutôt que de suggérer qu'il 
puisse s'agir d'un processus évolutif impliquant différents acteurs dans une trajectoire de vie.

C'est en tenant compte de ce contexte précis que l'on peut, d'une part, apprécier l'écart éventuel entre la pratique et l'esprit de la norme légale ${ }^{12}$ et, d'autre part, analyser la portée des demandes actuelles d'une plus grande ouverture. Mais encore fautil s'entendre sur les termes «ouverture» et «adoption ouverte».

\section{L'OUVERTURE: DÉFINITIONS ET PERCEPTIONS}

$\mathrm{Qu}^{\prime}$ entend-on exactement par «adoption ouverte»? Il n'entre pas dans notre propos de faire état ici des différentes pratiques d'ouverture qui existent actuellement au Québec. L'analyse des résultats de notre recherche sur cet aspect du sujet n'est pas terminée. Nous pouvons cependant affirmer, dès à présent, qu'il n'y a pas d'uniformité ni de définition unique au sein des différents services d'adoption et qu'il n'est pas rare de voir, dans un même service, des conceptions distinctes d'une professionnelle à une autre ${ }^{13}$. À titre d'exemple, pour certaines travailleuses sociales le simple fait qu'une mère puisse choisir le profil des parents adoptifs constitue en soi de l'adoption ouverte, parce qu'il s'agit d'une forme de contact, aussi indirect soit-il, et que la prise en considération de ce choix par le service d'adoption implique une certaine remise en question du modèle traditionnel. Par contre, pour d'autres, seul le maintien de contacts personnels directs post-adoption entre le parent biologique et l'enfant (ou ses parents adoptifs) est visé par le concept d'adoption ouverte, le reste ne constituant que des modalités mineures qui n'affectent pas réellement l'institution même de l'adoption.

L'expression «adoption ouverte» vient des États-Unis (open adoption) où elle est couramment utilisée depuis plus de vingt ans; son contenu y est cependant fluctuant ${ }^{14}$. En effet, la pratique révèle que la notion peut couvrir des degrés très variables de contacts entre parents d'origine et parents adoptifs, incluant ou non l'enfant: l'ouverture peut se limiter à un simple échange de renseignements, anonymes ou nominatifs, à des contacts avant ou pendant le processus d'adoption, à des contacts post-adoption sous toutes les formes (courrier direct ou indirect, en passant par l'intermédiaire des services d'adoption, contacts téléphoniques sporadiques ou soutenus, rencontres occasionnelles ou régulières, etc. ${ }^{15}$. À la limite, si l'on acceptait 
la définition la plus diluée (c'est-à-dire le simple échange de renseignements anonymes), il faudrait conclure qu'au Québec toutes les adoptions internes (par opposition à internationales) sont ouvertes, puisqu'en pratique elles donnent lieu de façon presque systématique à la transmission de données anonymes sur les antécédents, comme le prévoit d'ailleurs la loi. Demick et Wapner mentionnent une typologie des degrés d'ouverture: restricted open adoption (simple échange d'informations, de photos...), semi-open adoption (rencontre initiale entre les familles), full open adoption (contacts occasionnels et échanges de renseignements) et continuing open adoption (contacts continus, avec ou sans ententes formelles) ${ }^{16}$. D'un point de vue méthodologique, les acceptions divergentes de la notion, ainsi que les degrés tellement différents d'ouverture auxquels la littérature scientifique fait référence lorsqu'il est question d'adoption ouverte, rendent nécessaire une entente sur une définition opérationnelle ${ }^{17}$. À cet égard, on peut noter que les définitions proposées par la littérature nord-américaine sont basées sur le concept de communication entre les parties engagées dans le processus d'adoption. Seule l'intensité ou la fréquence de la communication peut changer.

Une définition basée sur le seul principe de la communication, à quelque degré que ce soit, nous est apparue insatisfaisante, car elle nous aurait obligés, dans la sélection des dossiers aux fins de la recherche, à considérer comme «ouvertes » à peu près toutes les adoptions tardives ainsi que toutes les adoptions par des familles d'accueil. En effet, dans la presque totalité de ces cas les familles se connaissent et ont, dès lors, entre elles un lien de communication. Pourtant, de toute évidence certaines de ces adoptions se sont réalisées dans un esprit de fermeture et selon le modèle traditionnel, c'est-à-dire avec un objectif clair d'évacuation complète du parent d'origine. C'est le cas dans la plupart des dossiers de déclaration d'admissibilité à l'adoption, c'est-à-dire des cas d'adoption sans le consentement des parents biologiques ${ }^{18}$. D'autre part, on constate que dans certains cas la mère biologique participe activement au choix des parents adoptifs sans qu'il y ait pour autant de communication ni de projet de maintien de liens quelconques entre eux. Or, il est incontestable que cette participation active de la mère constitue un élément d'ouverture par lequel le processus d'adoption s'écarte du modèle traditionnel ${ }^{19}$.

Afin d'inclure ce type d'ouverture tout en excluant les dossiers où, malgré certains liens préexistants entre les familles, 
l'adoption s'est déroulée dans un esprit de fermeture, nous avons opté pour une définition qui ajoute à la notion de communication celle de pouvoir. Selon nous, l'adoption ouverte vise dès lors non seulement celle où une communication est voulue, mais aussi celle qui offre la possibilité au parent biologique de s'approprier, dans une certaine mesure, le processus d'adoption et donc d'exercer un certain pouvoir sur celui-ci (en choisissant les adoptants; en formulant des exigences; en rencontrant les adoptants; en envoyant des lettres après le jugement, etc.). Ce n'est plus tant la communication que la participation des parties dans le processus qui définit alors l'élément d'ouverture. C'est donc à partir de cette définition qu'ont été sélectionnés les dossiers pour cette partie de la recherche.

Les discours entourant l'adoption ouverte sont souvent chargés émotivement: certains sont «pour» et d'autres sont «contre». Les deux positions traduisent des conceptions opposées de l'adoption et s'appuient, bien entendu, sur la notion de l'intérêt de l'enfant, les uns mettant l'accent sur les avantages de l'ouverture, les autres sur ses inconvénients. De nombreuses études font état des différents avantages possibles selon que l'on se situe du point de vue des parents adoptifs, de l'enfant ou du parent biologique ${ }^{20}$.

Par rapport à l'enfant, les arguments les plus courants (outre ceux reliés à la question de son identité généalogique) insistent, d'une part, sur la diminution de l'insécurité et de la croyance qu'a l'enfant d'avoir été rejeté par ses parents biologiques et, d'autre part, sur l'équilibre entre son besoin de permanence et son besoin de liens affectifs avec sa famille adoptive, ces liens étant libérés du traumatisme causé par la rupture des relations significatives avec la famille d'origine. Quant aux inconvénients les plus souvent cités, on peut retenir le risque d'interférence indue de la part du parent d'origine, le danger de conflits de loyauté et les problèmes d'identité pour l'enfant ${ }^{21}$. Il convient cependant de relever que bien des détracteurs de l'ouverture s'appuient essentiellement sur des impressions cliniques plutôt anecdotiques ou tout simplement sur des opinions personnelles.

En laissant de côté les discours rhétoriques sur l'adoption ouverte (et il y en a des deux côtés) pour ne retenir que les résultats des études empiriques rigoureuses sur le sujet, il est pourtant possible de faire un certain nombre de constats. Premièrement, on peut dire que pour le moment il n'existe pas 
d'études longitudinales significatives. Dès lors, la démonstration certaine des avantages ou inconvénients de l'ouverture, en ce qui concerne l'enfant, n'est pas faite à ce jour. Deuxièmement, on constate que le nombre d'études empiriques se multiplie depuis quelques années et que, mis à part quelques recherches de type exploratoire auprès d'un nombre trop faible de personnes pour être réellement significatives ${ }^{22}$, elles traduisent des points de convergence intéressants. Ainsi, la plupart des études font état d'un degré élevé de satisfaction des parents adoptifs à l'égard du type d'ouverture choisi ${ }^{23}$. Dans leur étude récente auprès de 190 familles adoptives et de 169 mères biologiques engagées dans des adoptions allant du plus fermé au plus ouvert, Grotevant et McRoy font notamment le constat suivant en ce qui concerne l'adoption à haut degré d'ouverture: les parents adoptifs font preuve de plus d'empathie à l'égard de l'enfant et de sa famille d'origine, leur sentiment de permanence dans leur relation avec l'enfant semble plus profond et ils ressentent moins la crainte de voir la mère biologique réclamer un jour leur enfant. Tout en soulignant que leurs résultats démentent ainsi les principales critiques généralement adressées à l'ouverture, ces chercheurs refusent cependant de voir dans l'adoption totalement ouverte la panacée universelle car, constatent-ils, le besoin et le degré d'ouverture fluctuent avec le temps. Il n'entre pas dans notre propos de faire le relevé de toutes les études recensées. Mentionnons simplement que la conclusion que l'on peut tirer de la revue de la littérature américaine concernant la perception de l'ouverture qu'ont les parties est généralement d'un optimisme prudent ${ }^{24}$.

\section{LA COLLECTE DES DONNÉES}

Au Québec, la question de l'adoption ouverte est sous-documentée. La présente étude, de type qualitatif et descriptif, entend donc amorcer la réflexion sur ce qui apparaît être une pratique de plus en plus courante ${ }^{25}$ ainsi que sur la signification de cette pratique au regard de la parentalité. Cette partie de notre recherche porte sur les dossiers d'adoption ouverte du Centre des services sociaux de Québec (maintenant Centres jeunesse de Québec) dans lesquels un jugement d'adoption fut prononcé entre janvier 1993 et juin 1995. À partir de la liste informatisée de tous les jugements d'adoption interne rendus sur le territoire de cette institution pendant la période visée, les intervenantes 
nous ont décrit brièvement les différents contextes d'adoption (rupture des contacts, processus clinique, nature des contacts, le cas échéant). Pour cette période de deux ans et demi nous avons dénombré 67 situations qui se profilent de la façon suivante: 38 adoptions ouvertes (dont quatre où «l'ouverture» se faisait à l'égard de grands-parents biologiques et deux à l'égard de membres de la parenté); 20 adoptions fermées; 9 adoptions par le conjoint du seul parent biologique. En excluant ce dernier cas, on peut retenir que, pour la période à l'étude, deux tiers des adoptions à Québec se sont réalisées dans un contexte d'ouverture.

Parmi les 38 situations initiales, les intervenantes ont repéré celles qui correspondaient à notre définition d'ouverture, c'est-à-dire celles où les parents biologiques (la mère le plus souvent) avaient participé au choix des adoptants ou maintenu certains contacts avec la famille adoptive. Dans un premier temps, c'est la professionnelle responsable de leur dossier d'adoption qui a communiqué avec les familles adoptives, afin de respecter la confidentialité des dossiers. Ce n'est qu'en cas de consentement donné à l'intervenante que les chercheurs ont ensuite pris le relais. Le nombre de refus de participer au projet fut minime; finalement, 30 dossiers ont pu être sélectionnés, ce qui représente la presque totalité des dossiers d'adoption ouverte pour la période retenue. La réalité de ces 30 enfants correspond à 26 situations familiales, car dans quatre cas deux enfants d'une même famille se retrouvent en situation d'adoption.

Deux démarches distinctes de collecte de données ont alors été effectuées. La première, pour laquelle les chercheurs ont été dûment autorisés par la Chambre de la jeunesse de la Cour du Québec, consistait en l'étude des dossiers des services d'adoption à l'aide d'une grille de collecte de données ayant fait l'objet d'un prétest. Il s'agit, avant tout, des dossiers d'adoption qui contiennent, outre le dossier judiciaire (requêtes, ordonnances, jugements), toutes les données cliniques (entrevues, questionnaires, rapports d'évaluation, historique du dossier) concernant l'enfant, la mère biologique (le père, le cas échéant) et les candidats à l'adoption. En plus des données purement descriptives, ces dossiers constituent une source d'information précieuse sur les motivations des parties en cause. Dans la plupart des cas, cette étape fut enrichie par l'étude d'un dossier de protection de l'enfant concerné. Les dossiers de protection ont permis de compléter les données sur la famille biologique. La deuxième démarche était constituée d'entrevues avec les parents adoptifs. 
Il s'agissait d'entrevues semi-structurées, d'une durée approximative d'une heure et demie à deux heures, au domicile des parents adoptifs ${ }^{26}$. Les entrevues ont été enregistrées et des sommaires ont ensuite été préparés pour chacun des thèmes abordés.

Bien que l'on puisse être porté à croire que l'adoption ouverte serait plutôt réservée à des enfants plus âgés, la réalité observée ici nous permet de constater que dans plus de la moitié (53\%) des situations, le jugement d'adoption avait été prononcé alors que les enfants avaient trois ans et moins. Une importante proportion des jugements (11 sur 30 ) ont été prononcés avant que l'enfant ait atteint l'âge de deux ans, et parmi ces enfants, sept étaient destinés à l'adoption dès leur naissance.

Selon les notes aux dossiers, les enfants étaient tous en bonne santé au moment de leur adoption sous réserve que deux d'entre eux présentaient des handicaps à la naissance (spinabifida et trisomie 21), lesquels constituent le motif même de leur adoption. On note également des problèmes d'apprentissage chez deux enfants. Près des deux tiers des enfants étaient par ailleurs suivis en protection de la jeunesse au moment de l'adoption, principalement pour des problèmes de négligence et d'abandon.

\section{PrÉSENTATION DES RÉSULtatS}

\section{Transfert de I'enfant vers la famille adoptive}

La majorité des mères étaient dans la vingtaine au moment de confier leur enfant en adoption. La réalité de la jeune mère adolescente n'est pas représentée ici. Par ailleurs, il s'agit surtout de mères seules ayant atteint un niveau secondaire de scolarité. Plus du tiers d'entre elles présentaient des problèmes importants, soit d'alcoolisme $(n=5)$, de toxicomanie $(n=8)$ ou de santé mentale $(n=5)$. La quasi-totalité de ces femmes sont bénéficiaires de l'aide sociale ou ont des revenus à peu près équivalents et vivent sans conjoint.

Une seule situation se distingue nettement de ce portrait. Il s'agit d'un couple se situant à un niveau supérieur de scolarité et qui a confié son enfant en adoption dès sa naissance en raison du fait qu'il était affecté d'une trisomie 21.

La moyenne d'âge des parents adoptifs se situe plus près de la quarantaine (mère : 39 ans et 3 mois / père : 39 ans et 8 mois). Il s'agit le plus souvent de couples mariés qui presque tous 
présentaient des problèmes de fertilité. Dans trois situations seulement, il s'agissait de femmes seules. On retrouve proportionnellement presque autant de parents ayant fait des études secondaires que de parents ayant poursuivi des études supérieures. La moitié des familles adoptives se retrouvent dans la classe de revenus de $50000 \$$ et plus. Abstraction faite de quelques cas, la situation typique est donc celle du transfert de l'enfant d'une famille monoparentale matricentrique à faible revenu vers un couple marié de classe moyenne.

Si l'on examine maintenant les changements que l'enfant a été amené à vivre sur le plan de la fratrie lorsqu'il est passé de sa famille d'origine à sa famille adoptive, on peut retenir ceci : la majorité $(70 \%)$ des enfants avaient des frères et sœurs dans le milieu d'origine au moment de l'adoption et près de $40 \%$ d'entre eux sont devenus enfants uniques dans la famille adoptive. À l'opposé, plus des deux tiers des enfants qui étaient seuls dans leur famille biologique se sont retrouvés avec des frères ou sœurs dans leur foyer d'adoption.

Les difficultés de vie du parent biologique constituent ici le principal motif d'adoption et, en ce sens, ces caractéristiques ne sont pas propres à une adoption dite ouverte puisqu'on retrouve des profils similaires chez des parents biologiques qui ont consenti à une adoption de type fermé. Comment se distinguent alors les contextes d'adoption ouverte?

\section{Différents contextes d'ouverture}

Chaque cas, bien entendu, a ses particularités et l'adoption renvoie par conséquent à différents scénarios. Dans les cas étudiés, nous en avons distingué quatre. Le premier scénario, qui correspond à $50 \%$ de nos situations, est celui de l'enfant placé en famille d'accueil et adopté par celle-ci après un certain temps de placement. L'âge de l'enfant varie entre 1 an et 11 ans au moment de son adoption.

Le deuxième scénario, qui concerne ici 9 enfants sur 30, est celui de l'enfant placé par les services sociaux dans une famille non connue des parents biologiques en vue de son adoption. Une proportion de ces familles étaient à la fois disponibles comme famille d'accueil et postulantes à l'adoption (banque mixte). Il s'agit surtout d'enfants de moins de trois ans (7 sur 9).

Le troisième scénario ( 4 cas sur 30 ) est celui de ces mères qui confient directement leur enfant à une famille de leur choix 
peu de temps après la naissance. Les adoptants se présentent après un certain temps pour faire légitimer l'adoption et le jugement d'adoption est prononcé avant que l'enfant ait atteint l'âge de deux ans.

Enfin, le dernier scénario (deux situations), qui se rapproche du précédent, est celui où des membres de la parenté biologique prennent temporairement en charge la garde de l'enfant et finissent par trouver dans leur entourage des personnes disposées à l'adopter.

Si l'on peut retenir, de ces quatre scénarios, que dans la plupart des cas l'enfant n'est mis en contact avec sa famille adoptive qu'une fois que celle-ci a été reconnue compétente par les services sociaux, il est tout de même intéressant de constater qu'un certain nombre de mères s'approprient directement le droit de choisir elles-mêmes la famille à qui elles confient leur enfant. Après écoulement du temps, il n'y a d'autre issue que d'officialiser ce choix au nom de l'intérêt de l'enfant. Une simple délégation de l'autorité parentale évolue ainsi, «par la force des choses» pourrait-on dire, en changement de filiation. Il s'agit bien de ce que certains qualifient $d^{\prime}$ «adoption privée » et que les normes légales entendent, en principe, prohiber.

Quant au motif le plus fréquemment évoqué par les mères biologiques pour consentir à l'adoption, l'examen des dossiers révèle qu'il s'agit du sentiment d'incompétence parentale et du désir de voir l'enfant pris en charge par des parents capables de donner ce qu'elles-mêmes ne peuvent offrir. Dans deux situations seulement, les mères ont choisi de faire adopter leur enfant comme solution alternative à un avortement expressément envisagé.

Il ne faut donc pas s'étonner qu'une forte proportion des mères (18 sur 26) manifestent explicitement des critères quant au choix des parents adoptifs. Ces critères correspondent en quelque sorte aux qualités que doit détenir un parent compétent selon la perception des mères biologiques. Mis à part les exigences relatives au maintien de contacts après l'adoption ou à la désignation des adoptants, les critères auxquels les mères adhèrent le plus fréquemment renvoient davantage au caractère normatif du rôle parental (tels les critères relatifs à l'âge, à la religion, à l'état civil...) plutôt qu'à des dimensions liées à l'environnement affectif (exemple: maison avec une cour, présence ou non d'autres enfants) dans lequel l'enfant aura à vivre. 


\section{TABleau 1}

Critères des mères quant au choix des adoptants

Critères normatifs

Favoriser des études supérieures

Valeurs conformes au social

État civil

Âge

Religion

Revenu

Profession

\section{Critères affectifs}

1 Favoriser les retrouvailles

2 Donner une bonne image

de la mère biologique

3 Présence d'autres enfants dans la famille adoptive

4 L'enfant doit rester enfant unique 1

5 Préserver la fratrie 1

5 Maison avec une cour $\quad 1$

6 Disponibilité affective

des parents adoptifs

Désignation des adoptants

Maintien de contacts

sous une forme ou une autre

Un certain nombre de mères ont exprimé leur souhait quant au maintien de liens avec l'enfant, alors que d'autres ne semblent pas avoir manifesté explicitement d'exigences à cet égard, même si dans les faits seule une faible minorité n'a plus aucun contact post-adoption (voir tableau 2).

\section{Modalités de contacts après le jugement d'adoption}

Les modalités d'ouverture privilégiées après le jugement d'adoption semblent fonction de la position des parents biologiques et adoptifs à l'égard de la confidentialité. Malgré l'idée du maintien des liens, plusieurs parents adoptifs décident de préserver leur anonymat. En conséquence, les modalités de contacts seront plutôt restreintes. En effet, comme le montre le tableau suivant, on constate que dans ces cas les contacts se limitent à l'échange par correspondance où l'intervenante du service d'adoption devient la principale intermédiaire (reçoit et achemine le courrier à bon port), ce qui permet de préserver la confidentialité. Par ailleurs, plus des deux tiers des parents biologiques et adoptifs choisissent de faire connaître leurs coordonnées réciproques. Les modalités de contacts sont alors plus diversifiées, allant de l'échange de correspondance au contact en face à face. 
TAbleau 2

Modalités et types de contacts après I'adoption

Types de contacts

\begin{tabular}{|c|c|c|}
\hline & $\begin{array}{l}\text { Nominaux } \\
(\mathrm{n}=21)\end{array}$ & $\begin{array}{l}\text { Anonymes } \\
\qquad(\mathrm{n}=9)\end{array}$ \\
\hline Rencontres & 12 & 0 \\
\hline Appels téléphoniques & 6 & 0 \\
\hline Cartes - Lettres & 3 & 8 \\
\hline Envoi de photos & 3 & 6 \\
\hline Contact interrompu ${ }^{*}$ & 3 & 1 \\
\hline Modalité inconnue $^{\star \star}$ & 2 & \\
\hline \multicolumn{3}{|c|}{$\begin{array}{l}\text { * Ces quelques cas s'expliquent par la définition que nous avons donnée de l'ouverture et qui } \\
\text { inclut les cas où la mère biologique participe activement au processus clinique, par exemple } \\
\text { en choisissant le profil ou l'identité des candidats adoptants. }\end{array}$} \\
\hline \multicolumn{3}{|c|}{$\begin{array}{l}\text { * Il s'agit de deux cas pour lesquels le dossier au service d'adoption révèle le maintien de } \\
\text { contacts mais où il nous a été impossible de vérifier la nature exacte des contacts en raison } \\
\text { du fait que ces parents ont finalement renoncé à accorder une entrevue. }\end{array}$} \\
\hline
\end{tabular}

Les modalités de contacts nous informent indirectement sur les modes de parentalité choisis par l'intermédiaire de cette pratique d'adoption ouverte. On voit ainsi se profiler deux types de parentalité: d'une part, une qui se maintient dans la tradition de l'adoption, c'est-à-dire une parentalité de substitution où les parents biologiques n'ont aucune place dans le quotidien de l'enfant; d'autre part, une parentalité additionnelle où les parents adoptifs pourraient éventuellement reconnaître aux parents biologiques certaines fonctions parentales qui ne font cependant pas appel à l'autorité parentale, tel le gardiennage.

\section{Perception des parents adoptifs}

Les avantages et les inconvénients exprimés par les parents adoptifs à l'égard de l'adoption ouverte portent sur deux niveaux de parentalité. D'une part, lorsqu'ils expliquent les avantages, c'est essentiellement du droit de l'enfant à ses origines, à son identité, à son histoire qu'il est question. Par contre, lorsqu'ils mentionnent certains inconvénients, les énoncés renvoient davantage au lien affectif qui pourrait être entretenu entre le parent biologique et l'enfant. Les parents adoptifs veulent préserver des traces de l'histoire de l'enfant et reconnaissent 
de façon assez unanime l'importance de ne pas faire de secret sur le passé de l'enfant. Cependant, ils sont généralement loin d'imaginer que des relations quotidiennes - ou même régulières - entre le parent biologique et l'enfant pourraient être possibles.

À cet égard, on peut noter chez la plupart des parents un sensible changement d'attitude une fois que le jugement d'adoption a été prononcé. Avant le jugement, c'est-à-dire lorsqu'ils ne sont que candidats à l'adoption, les futurs adoptants se montrent généralement sensibles à l'idée d'une possible ouverture post-adoption. Cette éventualité leur est d'ailleurs formellement présentée par le CPEJ comme une composante de l'adoption contemporaine. Chez certains candidats, on peut même noter un assouplissement des exigences au fil du temps lorsqu'ils sont en attente d'un enfant depuis plusieurs années. Par exemple, dans un dossier où les candidats avaient exprimé initialement leur préférence pour un bébé de six mois et pour une adoption fermée, ils acceptent, au bout de trois ans d'attente, l'idée d'accueillir un enfant plus âgé. Finalement, ils se disent prêts à un type très ouvert d'adoption après avoir reçu du centre des services sociaux une lettre les avisant que l'ouverture fait désormais partie du portrait de l'adoption et leur demandant s'ils sont toujours intéressés par le projet à ces conditions.

Une fois le jugement prononcé, les parents adoptifs font valoir leur priorité comme parents quant aux décisions à prendre concernant l'enfant. En entrevue, ils reconnaissent que durant le processus clinique le parent biologique a le pouvoir de refuser l'adoption en leur faveur. Mais une fois le jugement d'adoption prononcé, ils ont plutôt un discours d'appropriation du rôle parental; le contrôle de la situation leur appartient désormais. Dans le discours, ce changement est légitimé par l'argument de l'intérêt évolutif de l'enfant. L'extrait d'entrevue qui suit est représentatif de cette position:

J'ai eu peur à un moment donné quand on a fait la clarification du projet de vie et qu'on a essayé que la petite aille revivre avec sa mère. [...] C'est à moi de décider; au début j'essayais de respecter notre entente mais j'ai vu que la petite était perturbée. On se parle au téléphone, la mère et moi. C'est mieux comme ça pour maintenant; plus tard on verra.

Certains expriment avoir vécu une période plutôt stressante lorsque l'ordonnance de placement de l'enfant, en vue de son adoption, était en cours, puisque le parent biologique pouvait à 
tout moment reprendre son enfant ou encore disqualifier les candidats comme parents adoptifs. Après l'adoption, on assiste donc à une appropriation du pouvoir par les parents adoptifs sur le devenir de l'enfant et sur les contacts (et leur nature) avec les parents biologiques, comme l'illustrent les propos de ce parent:

Les parents ont eu leur chance de démontrer qu'ils voulaient garder leur enfant. Ils ne l'ont pas fait. [...] Si ça perturbe l'enfant de revoir son père, je suis le père maintenant et j'ai le droit de décider si ce n'est pas bon pour lui. Comme je le ferais si c'était une autre personne que le parent que je ne voudrais pas que mon enfant fréquente.

Tous les parents adoptifs expriment d'ailleurs la conviction que leur autorité parentale implique le droit de décider du maintien ou de la rupture des contacts, au nom de l'intérêt de l'enfant. Il n'est donc pas surprenant que malgré la crainte exprimée par certains de voir leur autorité menacée par l'éventuelle présence de la mère biologique $(n=7)$, plus des deux tiers des parents adoptifs se disent favorables à l'adoption ouverte, mais dans les limites qu'ils se sont données, que ce soit en permettant à l'enfant de rencontrer son parent biologique ou d'en avoir des nouvelles. Cependant, les parents qui ont opté pour un maintien de contact qui se limite à l'échange ponctuel de renseignements anonymes soulignent l'importance de cette ouverture, mais estiment que l'anonymat est une garantie essentielle contre la fragilisation de leur statut parental.

\section{DISCUSSION}

Tout en considérant les limites de cette étude qui n'a pas la prétention de représenter la réalité globale de l'adoption ouverte, nous souhaitons faire état ici de quelques réflexions auxquelles cette première démarche empirique nous amène.

Les situations d'adoption ouverte observées s'inscrivent dans une trajectoire de services en protection de la jeunesse où une bonne part des enfants transitent d'abord par une étape de placement en famille d'accueil avant que se concrétise le projet d'adoption. Cela a pour effet d'inscrire l'adoption dans un processus ouvert en soi qui n'a pas nécessairement d'effet sur la disposition des parents adoptifs et des parents biologiques à maintenir des contacts après le jugement d'adoption. Si pour la plupart des parents adoptifs «l'ouverture» constitue un droit de l'enfant - et pour certains même un droit du parent biologique - 
tous, comme nous avons pu le constater, subordonnent l'exercice de ce droit à leur propre contrôle. Ils se sentent confortés dans cette position par le service d'adoption qui leur reconnaît le droit d'exercer leur autorité parentale ainsi que le prévoient les normes légales. Tout au plus le service d'adoption interviendra-t-il, à la demande d'une mère biologique, pour rappeler aux parents adoptifs les termes de l'entente «morale» qu'ils ont passée avec la mère de l'enfant, lorsque, par exemple, ils négligent d'envoyer des nouvelles aux dates prévues. L'entente n'a pas de valeur coercitive, ni dans l'esprit des parents adoptifs, ni dans celui des intervenantes.

L'adoption ouverte remet-elle dès lors en question la conception classique de la parentalité? À première vue, la pratique de l'adoption ouverte pourrait en effet sous-entendre un nouveau mode de parentalité où les parents adoptifs peuvent réserver certaines fonctions parentales aux parents biologiques s'apparentant aux droits que conserve un parent non gardien lors d'une séparation ou d'un divorce. Notre analyse nous permet cependant d'avancer que le mode qui prévaut le plus souvent demeure celui de la parentalité de substitution. Ce n'est qu'exceptionnellement que l'on voit apparaître l'idée d'une parentalité additionnelle et dans des contextes très particuliers, par exemple lorsque l'enfant est âgé et qu'il a passé quelques années avec ses parents ou qu'il s'agit des grands-parents qui veulent s'impliquer activement dans la vie de l'enfant.

En dehors de la justification clinique, on peut se demander comment les parents adoptifs peuvent adhérer à un tel projet s'ils n'ont pas d'intérêt eux-mêmes à partager leurs préoccupations autour de l'enfant avec les parents biologiques. Cet engagement nous apparaît s'articuler autour du «droit futur aux origines " (plus tard mon enfant pourra éventuellement renouer avec sa famille biologique). En effet, ce droit de l'enfant devient la pierre angulaire du discours dominant des parents quant à leur adhésion à l'adoption ouverte. Les entrevues avec les parents adoptifs nous révèlent que les retrouvailles entre les parents biologiques et l'enfant sont présentées comme un élément faisant partie du projet d'adoption. Ainsi, l'adoption ouverte s'inscrit dans cette même logique de la reconnaissance de la réalité biologique de l'enfant comme une dimension importante à considérer dans la construction de son identité. Le maintien de certains liens, et donc l'idée d'ouverture, est alors envisagée "en prévision de...» plutôt que comme modus vivendi. Cela nous 
permet de comprendre que certains parents s'en tiennent à des contacts anonymes, en passant par des services d'adoption. En ce sens, paradoxalement, la confidentialité n'est donc pas toujours un obstacle à l'adoption dite ouverte. L'idée d'ouverture en adoption renvoie, il est vrai, à une possibilité de maintien des contacts entre les parents biologiques et l'enfant, mais n'évacue pas pour autant la question de l'anonymat des parties qui était l'un des fondements de l'adoption dans sa forme traditionnelle.

Cette étude permet de constater que nous sommes loin du mythe de l'adoption ouverte comme pratique qui permet de conserver l'ensemble des liens significatifs dans la vie de l'enfant et donc d'envisager l'idée d'une parentalité additionnelle. Loin aussi de certaines présentations enthousiastes de l'ouverture ou de la définition de l'adoption proposée par Silber et Speedlin: "Adoption is the process of accepting the responsability of raising an individual who has two sets of parents ${ }^{27} »$. Certes, l'adoption ouverte, telle que nous l'avons approchée ici, se présente comme une réalité à peu près incontournable à laquelle les candidats à l'adoption se voient confrontés. Le processus clinique les met en position d'être choisis ou non par les parents biologiques qui consentent à l'adoption de leur enfant selon certaines exigences dont on a vu qu'elles pouvaient être très précises. Mais, sauf exception pour quelques adoptions (notamment d'enfants plus âgés), une fois que le jugement est prononcé, le modèle classique de parentalité de substitution s'impose tout en s'accommodant de quelques parenthèses dans la vie de l'enfant, histoire de ne pas perdre les traces de ceux qui l'ont mis au monde.

En conclusion, il importe de souligner que les enjeux de l'adoption ouverte ne devraient être ni sous-estimés, ni surestimés.

Plusieurs raisons devraient nous inciter à ne pas sousestimer la signification de l'adoption ouverte. Au stade clinique d'abord, car l'ouverture constitue une appropriation évidente du processus d'adoption au profit des mères biologiques. La possibilité de choisir l'identité des adoptants constitue l'expression la plus radicale de cette réalité. De plus, la reconnaissance de l'adoption privée, sous réserve du contrôle étatique de l'intérêt de l'enfant, constitue une remise en question claire du système actuel de consentement général. D'autre part, au stade postjugement, l'acceptation d'une possibilité de maintien de liens directs et personnels entre l'enfant et le parent biologique bouleverse radicalement l'esprit et la lettre de l'adoption tradition- 
nelle. Le fait que dans la majorité des cas l'enfant avait une fratrie avant l'adoption est une réalité qui prend ici toute sa signification, car le maintien de liens pourrait également viser la fratrie d'origine et celle à venir. Le simple fait que cette possibilité d'ouverture puisse être envisagée, que ce soit pour un enfant plus âgé ou pour un nourrisson, constitue en soi une mutation majeure de l'adoption contemporaine.

L'adoption ouverte ne devrait cependant pas être surestimée non plus car, comme nous l'avons vu dans la plupart des cas étudiés, elle aboutit à la mise en place d'une parentalité de substitution, ce qui nous rapproche sensiblement du modèle classique. Pour ceux-là, il se pourrait bien que l'adoption ouverte ne soit pas un «nouveau modèle d'adoption », mais plus simplement "une autre façon de faire »,qui s'inscrit dans le même paradigme que l'adoption traditionnelle.

\section{Notes}

1. N.F. Belbas (1987), "Staying in Touch : Empathy in Open Adoption», Smith College Studies in Social Work, $\mathrm{n}^{\circ}$ 57, p. 184.

2. Il faut noter, cependant, que dans certains pays de tradition civiliste, comme la France ou la Belgique, il existe plusieurs formes d'adoption dont l'adoption simple qui laisse subsister, après l'adoption, certains liens entre l'enfant et sa famille biologique, notamment en ce qui concerne les droits successoraux.

3. F.-R. Ouellette note que les intérêts personnels des postulants à l'adoption (exemple : résoudre un problème de fertilité ou transmettre son nom) fragilisent leur position, particulièrement dans le cadre de l'évaluation psychosociale à laquelle ils doivent se soumettre («Redéfinitions de l'enfant et de la famille : la problématique généalogique en adoption», dans IQRC (1996), Enfances. Perspectives sociales et pluriculturelles, sous la direction de R.B. Dandurand, R. Hurtubise et C. Le Bourdais, Sainte-Foy: PUL, p. 81, 84).

4. H.D. Kirk (1964), Shared Fate, New York: The Free Press et, plus récemment, (1981) Adoptive Kinship, Toronto : Butterworths.

5. Voir par exemple l'étude souvent citée de A. Baran, R. Pannor et A.D. Sorosky (1974), «Adoptive Parents and the Sealed Record Controversy », Social Casework, $\mathrm{n}^{\circ}$ 531, p. 55. Des mêmes auteurs, voir aussi (1989), The Adoption Triangle. Sealed or Open Records : How They Affect Adoptees, Birth Parents, and Adoptive Parents, San Antonio : Corona Publ. Co.

6. Il n'entre pas dans notre propos de revenir sur les facteurs d'ordre démographique ou socioculturel qui ont radicalement changé la face même de l'adoption et qui ont été amplement décrits par la littérature scientifique (dénatalité, progression de la famille monoparentale, acceptation sociale du statut d'enfant «naturel», etc.). Voir notamment D.H. Siegel (1993), "Open Adoption of Infants : Adoptive Parent's Perceptions of Advantages and Disadvantages », Social Work, n ${ }^{\circ} 15$, p. 38. 
7. Voir notamment les articles suivants : A.R. Appell (1995), «Blending Families through Adoption : Implications for Collaborative Adoption Law and Practice», B.U.L. Rev., n ${ }^{\circ} 997$, p. 75 ; C.E. Cordle (1995), «Open Adoption: The Need for Legislative Action», Va. J. Soc. Pol'y \& L., no 275, p. 2 ; N.E. Dowd (1994), «A Feminist Analysis of Adoption », compte rendu de Family Bonds: Adoption and the Politics of Parenting, de Elizabeth Bartholet, Boston et New York: Houghton Mifflin, 1993, Harv. L. Rev., no 107, p. 913.

8. À noter que depuis l'entrée en vigueur ( $1^{\text {er }}$ janvier 1994$)$ du nouveau Code civil du Québec, les retrouvailles deviennent possibles, à certaines conditions, dès que l'enfant a atteint l'âge de 14 ans.

9. La loi donne une définition restrictive de proche parent; il s'agit des ascendants de l'enfant (grands-parents), de ses oncles et tantes et de leurs conjoints et, finalement, du conjoint du parent biologique (art. 555 du Code civil du Québec).

10. Art. 131.1 et 131.2 de la Loi sur la protection de la jeunesse, LRQ, c. P-34.1.

11. Art. 2.4 de la Loi sur la protection de la jeunesse, LRQ, c. P-34.1.

12. Cela ne signifie pas que la pratique soit «illégale», mais simplement qu'elle propose une autre approche de l'adoption. Il restera à voir dans quelle mesure la pratique peut continuer à se contenter du cadre juridique actuel ou si, au contraire, elle entraînera un réajustement des normes légales comme cela s'est produit déjà dans d'autres pays et dans d'autres provinces. L'importance des enjeux juridiques de l'ouverture explique peut-être pourquoi, à ce jour au Canada, ce sont surtout les juristes qui se sont intéressés à l'adoption ouverte (voir M. Bernstein, D. Caldwell, G.B. Clark et R. Zisman (1992), « Adoption with Access or "Open Adoption" », Can. Fam. Law Q., n ${ }^{\circ} 283$, p. 8 et J. House (1996), "The Changing Face of Adoption: The Challenge of Open and Custom Adoption », Can. Fam. Law Q., n 13, p. 333.

13. Nous utilisons le féminin, car la très grande majorité des personnes œuvrant dans les services d'adoption au Québec sont des femmes.

14. La première définition recensée date de 1976 et est souvent citée: An open adoption is one in which the birth parents meet the adoptive parents, participate in the separation and placement process, relinquish all legal, moral, and nurturing rights to the child, but retain the right to continuing contact and to knowledge of the child's whereabouts and welfare (A. Baran, R. Pannor et A.D. Sorosky (1976), «Open adoption", Social Work, $\mathrm{n}^{\circ} 21$, p. 97-100).

15. M. Berry (1991), «The practice of open adoption : findings from a study of 1396 adoptive families », Child and Youth Services Review, n ${ }^{\circ}$ 13, p. 379-395.

16. J. Demick et S. Wapner (1988), «Open and Closed Adoption : a Developmental Conceptualization », Family Process, n 229 , p. 27. Voir également C. Leblanc et D. Proulx, «L'adoption ouverte: conséquences sur l'enfant et ses parents » dans MSSS (1994), Colloque Adoption 94. Dessine-moi une famille, Québec, p. 96-109.

17. Sur l'urgence de définir la notion d'adoption ouverte, voir H.D. KIRK (1995), Looking Back, Looking Forward. An Adoptive Father's Sociological Testament, Indianapolis: Perspectives Press, p. 25.

18. Il peut cependant arriver que l'adoption ouverte soit considérée comme appropriée, même dans des cas de déclaration d'adoptabilité. En ce sens, voir J. Larin (1993), Normes Adoption, volet 1 : l'adoptabilité, Montréal, Les Centres jeunesse de Montréal, p. 34.

19. Dans les documents du ministère de la Santé et des Services sociaux, la notion d'adoption ouverte vise d'ailleurs essentiellement l'adoption privée, c'est-à-dire celle où les adoptants ont été choisis par la mère biologique. Voir MSSS (1994), L'adoption: un projet de vie. Québec. 
20. L.A. Ames (1992), "Open Adoptions: Truth and Consequences », Law \& Psychology Review, n 16, p. 137 ; C. Amadio et S.L. Deutsch (1983-1984), « Open adoption : allowing adopted children to "stay in touch" with blood relatives", Journal of Family Law, $\mathrm{n}^{\circ} 22$, p. 59; M. Berry (1991), "The effects of open adoption on biological and adoptive parents and the children: the arguments and the evidence», Child Welfare, n ${ }^{\circ}$ 70, p. 637-651; M. Berry (1993), "Adoptive parents' perceptions of, and comfort with, open adoption », Child Welfare, $\mathrm{n}^{\circ} 72$, p. 231-253 ; M. Berry (1991), «The practice of open adoption : findings from a study of 1396 adoptive families », Child and Youth Services Review, $\mathrm{n}^{\circ} 13, \mathrm{p} .379$ 395 ; J. Etter (1993), "Levels of cooperation and satisfaction in 56 open adoptions», Child Welfare, n ${ }^{\circ} 72$, p. 257-267; R.G. Mc Roy, H.D. Grotevant et K.L. White (1988), Openness in adoption: new practices, new issues, New York: Praeger Publishers; D.H. Siegel (1993), «Open Adoption of Infants : Adoptive Parents' Perceptions of Advantages and Disadvantages », Social Work, n ${ }^{\circ} 38$, p. 15 ; R.P. Barth (1987), "Adolescent Mothers' Beliefs About Open Adoption », Social Casework, p. 323.

21. A.D. Byrd (1988), «The case for confidential adoption », Public Welfare, vol. 46, $\mathrm{n}^{\circ} 4$, p. 20-23 ; A.D. Kraft et al. (1985), "Some theoretical considerations on confidential adoptions. Part III : The adopted child», Child and Adolescent Social Work Journal, no 2, p. 139-153 ; A.D. Kraft et al. (1986), «Some theoretical considerations on confidential adoptions. Part IV : Countertransference», Child and Adolescent Social Work Journal, $\mathrm{n}^{\circ} 3$, p. 3-14.

22. Par exemple, N.F. Belbas (1987), "Staying in Touch: Empathy in Open Adoptions », Smith College Studies in Social Work, no 57, p. 184-198 ; R.G. Mc Roy, H.D. Grotevant et K.L. White (1988), Openness in adoption: new practices, new issues, New York: Praeger Publishers.

23. J. Etter (1993), «Levels of cooperation and satisfaction in 56 open adoptions", Child Welfare, no 72, p. 257-267 ; M. Berry (1993), "Adoptive parents' perceptions of, and comfort with, open adoption », Child Welfare, $\mathrm{n}^{\circ} 72$, p. 231253.

24. H.E. Gross (1993), «Open Adoption: A Research-Based Literature Review and New Data », Child Welfare, $\mathrm{n}^{\circ} 72$, p. 269.

25. MSSS (1994), L'adoption: un projet de vie, Québec.

26. Quatre familles ont cependant opté pour une entrevue téléphonique, alors qu'une famille a préféré répondre à un questionnaire écrit et que deux familles ont finalement renoncé à participer à l'entrevue. Pour ces deux derniers cas, nous nous sommes donc contentés de la collecte des renseignements aux dossiers du service d'adoption.

27. K. Silber et P. Speedlin (1991), Dear Birthmother , 2e éd., San Antonio, Texas : Corona Publ. Co., p. 141. 
